\title{
Bisdemethoxycurcumin exerts a cell-protective effect via JAK2/STAT3 signaling in a rotenone-induced Parkinson's disease model in vitro
}

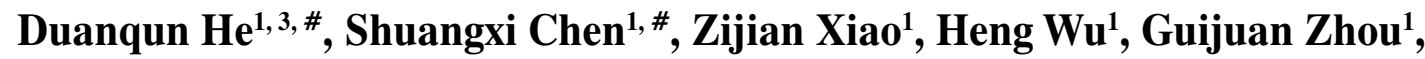 \\ Chenlin $\mathrm{Xu}^{1}$, Yunqian Chang${ }^{1}$, Yihui $\mathrm{Li}^{1}$, Gang Wang ${ }^{2, *}$, Ming Xie ${ }^{1, *}$
}

${ }^{1}$ The First Affiliated Hospital, University of South China, Hengyang, Hunan 421001, PR China

${ }^{2}$ Department of Rehabilitation, Union Hospital, Tongji Medical College, Huazhong University of Science and Technology, Wuhan, Hubei 430022, PR China

${ }^{3}$ Shenzhen Baoan People's Hospital (Group), The Second People's Hospital, Shenzhen, Guangdong 518101, China

\#These authors contributed equally to this study.

\begin{abstract}
Introduction. Oxidative stress and cell apoptosis have both been suggested to be closely associated with the pathogenesis of Parkinson's disease (PD). Previously, bisdemethoxycurcumin (BDMC) has been shown to exhibit several desirable characteristics as a candidate neuroprotective agent, including antioxidant and anti-inflammatory activities in the nervous system. However, whether BDMC can exert cell-protective roles in an in vitro model of PD remains unknown.

Material and methods. SH-SY5Y cells were pretreated with BDMC, with or without AG490 and SI-201, for 30 $\mathrm{min}$, followed by a co-incubation with rotenone for $24 \mathrm{~h}$. Subsequently, a cell viability assay and western blotting was performed, and SOD and GSH activities were analyzed.

Results. The results revealed that the pretreatment with BDMC enhanced the cell survival, antioxidative stress capacity and the phosphorylation levels of JAK/STAT3 in SH-SY5Y cells treated with rotenone. However, following the incubation with AG490 and SI-201, inhibitors of the JAK/STAT3 signaling pathway, BDMC was unable to exert cell-protective roles in SH-SY5Y cells treated with rotenone.

Conclusions. In conclusion, the results suggested that BDMC may exert a cell-protective role in SH-SY5Y cells in vitro via JAK2/STAT3 signaling, thus suggesting the possible application of BDMC for the treatment of neurodegenerative diseases related to JAK2/STAT3 signaling. (Folia Histochemica et Cytobiologica 2020, Vol. 58, No. 2, 127-134)
\end{abstract}

Key words: oxidative stress; rotenone; SH-SY5Y cells; bisdemethoxycurcumin (BDMC); JAK/STAT3

\section{Introduction}

Parkinson's disease (PD), a common neurodegenerative disease [1], is mainly characterized by a substantial loss of dopaminergic neurons in the substantia nigra, which causes a reduction of striata dopamine,

Correspondence address: Ming Xie and Gang Wang, e-mail: c237423281@163.com; wangzuogang@21cn.com and promotes cognitive impairment and functional defects [2]. Both motor and non-motor symptoms are observed in patients with $\mathrm{PD}$, who require assistance in performing all activities [3, 4]. It has been suggested that the excessive generation of reactive oxygen species (ROS), oxidative stress, neuroinflammation and mitochondrial dysfunction may all account for the loss of dopaminergic neurons and neuronal apoptosis [57]. However, the available current therapeutic strategies only provide symptomatic improvements due to the complex pathogenesis of PD. As a consequence, 
there remains an urgent requirement to determine the pathological mechanisms of PD to discover novel potential therapeutic targets to effectively treat PD.

Bisdemethoxycurcumin (BDMC), a natural derivative of curcumin, has demonstrated higher polarity, hydrophilicity and water solubility compared with the parent compound curcumin [8]. It was also reported to exert considerable antioxidant, anti-inflammatory and antiproliferative activities [9], and it demonstrated more effective pharmacological properties than curcumin [10], which suggested that it may be a more promising drug for clinical use [11].

Oxidative stress is considered to be the main cause of dopaminergic neuron degeneration in $\operatorname{PD}[12,13]$. Attempts to target signaling pathways that may be associated with the pathological process of PD have been made to counteract oxidative stress and cell apoptosis, of which one of the signaling pathways is the Janus-activated kinase 2/signal transducer and activator of transcription 3 (JAK2/STAT3) signaling pathway. Briefly, the signaling pathway consists of ligands binding to the membrane receptor and inducing the formation of Janus-activated kinase 2 (JAK2) binding sites in the cytoplasm to phosphorylate JAK2. Subsequently, the exposure of the STAT3 anchor point leads to its phosphorylation and activation, resulting in the translocation of STAT3 from the cytoplasm to the nucleus, to promote the transcription and expression of target genes [11]. Phosphorylated STAT3 has been discovered to promote cell proliferation, anti-inflammatory properties and the inhibition of apoptosis [14], and it also exerted neurotrophic effects on newly generated neurons and synapses [15].

The aim of the present study was to investigate the effects and underlying mechanisms of BDMC in SH-SY5Y cells treated with rotenone, an agent used to mimic the behavioral and neuropathological conditions of PD. The results indicated that BDMC may exert a cell-protective role against rotenone-induced neurotoxicity in SH-SY5Y cells via JAK2/STAT3 signaling.

\section{Materials and methods}

BDMC. BDMC, which contains $>80 \%$ curcumin and $>94 \%$ curcuminoid content purity, was purchased from Sigma-Aldrich (St. Louis, MO, USA). For cell incubations, BDMC was dissolved in $0.2 \%$ DMSO.

Cell culture and treatments. SH-SY5Y cells were cultured as previously described [16]. It was previously reported that BDMC exerted protective effects against cytotoxicity in retinal pigment epithelial cells at a concentration of
$15 \mu \mathrm{M}$ [17]. Also, $100 \mathrm{nM}$ rotenone induced neuronal loss, neuroinflammation, oxidative stress and NF- $\kappa$ B activity [18]. Therefore, the present study selected $15 \mu \mathrm{M}$ BMDC (the protective concentration) and $100 \mathrm{nM}$ rotenone (the neurotoxic concentration) for subsequent experiments. A total of $1 \times 10^{4} \mathrm{SH}-\mathrm{SY} 5 \mathrm{Y}$ cells were seeded into 96-well cell culture plates (for the cell viability assay) or 24-well cell culture plates (for SOD, GSH and western blotting assays), and treated as follows: 1) Cells were pretreated with $15 \mu \mathrm{M}$ $\mathrm{BDMC}$ for $30 \mathrm{~min}$ prior to a 24-h co-culture with $100 \mathrm{nM}$ rotenone; 2) cells were pretreated with $10 \mu \mathrm{M}$ AG490 and $15 \mu \mathrm{M}$ BDMC for 30 min prior to a $24-\mathrm{h}$ co-culture with rotenone; or 3) cells were pretreated with $100 \mathrm{nM} \mathrm{S3I-201}$ and $15 \mu \mathrm{M}$ BDMC for $30 \mathrm{~min}$ prior to a $24-\mathrm{h}$ co-culture with rotenone. At the end of the treatment duration, a cell viability assay and western blotting were performed, and SOD and GSH activities were analyzed.

Cell viability assay. Cell viability assay was performed as previously described [19-21], with minor modifications. Briefly, at the indicated time points, SH-SY5Y cells were cultured in DMEM, supplemented with $10 \mu \mathrm{l}$ 3-(4, 5-dimethyl-2-thiazolyl)-2, 5-diphenyl- 2-H-tetrazolium bromide (MTT; $500 \mu \mathrm{g} / \mathrm{ml}$; cat. no. M1020; Beijing Solarbio Science \& Technology, Co., Ltd., Shanghai, China) for $4 \mathrm{~h}$. After aspirating the culture medium, $100 \mu$ l dimethyl sulfoxide (DMSO) was added to each well in the culture plates and the cells were incubated at $37^{\circ} \mathrm{C}$ for $30 \mathrm{~min}$. Finally, the optical density was measured with a spectrophotometer at a wavelength of $410 \mathrm{~nm}$.

Measurement of SOD activity. The WST-1 Cell Proliferation assay kit was used to detect SOD activity, according to the manufacturer's instructions (cat. no. A001-3-2; Jiancheng Biotech Ltd., Nanjing, China) [22, 23]. The xanthine-xanthine oxidase system was applied to produce superoxide ions, which can react with 2-(4-iodophenyl)-3-(4-nitrophenol-5-phenlyltetrazolium chloride) to form a red formazan dye. The absorbance was determined at the wavelength of $550 \mathrm{~nm}$. The protein concentration was determined using a BCA protein assay kit (QPBCA, Sigma-Aldrich; Merck KGaA, Saint Louis, MO, USA). The values were expressed as units/mg protein, with one unit of SOD defined as the amount of SOD inhibiting the rate of reaction by $50 \%$ at $25^{\circ} \mathrm{C}$.

Measurement of GSH. Measurement of GSH content was performed according to a previously described method [24], with minor modifications. Briefly, SH-SY5Y cells in the culture medium were centrifuged at $500 \times g$ for $10 \mathrm{~min}$ and washed with PBS twice. The collected SH-SY5Y cells were resuspended in protein removal reagent and vigorously vortexed. Subsequently, the samples were frozen rapidly and thawed with liquid nitrogen twice at $37^{\circ} \mathrm{C}$, and then incubated at $4^{\circ} \mathrm{C}$ for $5 \mathrm{~min}$. Following centrifugation at $10,000 \times g$ for $10 \mathrm{~min}$, the supernatants were collected. The 
determination of GSH levels was performed using a GSH and GSSG assay kit (cat. no. S0053; Beyotime Institute of Biotechnology, Shanghai, China), according to the manufacturer's instructions.

Western blotting. Western blotting was performed as previously described [25-27], with minor modifications. Briefly, whole cell lysates were obtained using a RIPA lysis buffer mixture (Beijing Solarbio Science \& Technology Co., Ltd.), supplemented with PMSF (1:200; Beijing Solarbio Science \& Technology Co., Ltd.). The lysates were then combined with 25\% LDS Sample buffer (Invitrogen; Thermo Fisher Scientific, Inc., Carlsbad, CA, USA) and heated at $95^{\circ} \mathrm{C}$ for $15 \mathrm{~min}$. Protein samples were separated via $10 \%$ SDS-PAGE and transferred onto polyvinylidene difluoride (PVDF) membranes (EMD Millipore, Billerica, MA, USA) for $3 \mathrm{~h}$ at $300 \mathrm{~mA}$. The membranes were blocked with 5\% nonfat dry milk or BSA dissolved in Tris- $\mathrm{HCl}$ saline buffer containing 0.1\% Tween-20 (TBST; pH 7.4). Subsequently, the blots were incubated overnight at $4^{\circ} \mathrm{C}$ with the following antibodies: Rabbit anti-p-STAT3 (1:500; cat. no. S2690; Sigma-Aldrich; Merck KGaA) and rabbit anti-p-JAK2 (1:500; cat. no. SAB4300124; Sigma-Aldrich; Merck KGaA). Following the primary antibody incubation, the membranes were washed three times for $5 \mathrm{~min}$ each in TBST and incubated with HRP-conjugated goat anti-rabbit secondary antibodies (1:1,000; Boster Biological Technology, Wuhan, China) diluted in TBST for $1 \mathrm{~h}$. The membranes were washed three times in TBST for 5 min each at room temperature and proteins bands were visualized with an enhanced chemiluminescence (ECL) solution (Bio-Rad Laboratories, Inc., Hercules, CA, USA). The expression levels were semi-quantified using ImageJ 5.0 software (National Institutes of Health, Bethesda, MD, USA).

Statistical analysis. Data are presented as the mean \pm SEM. Statistical comparisons between groups were performed using a Student's $t$ test for independent samples on SPSS 18.0 software (SPSS, Inc., New York, NY, USA). P $<0.05$ was considered to indicate a statistically significant difference.

\section{Results}

\section{BDMC treatment enhances cell survival and the antioxidative stress ability in SH-SY5Y cells treated with rotenone}

To investigate the effects of BDMC treatment on rotenone-induced SH-SY5Y cells, a cell viability assay was performed, and SOD and GSH activities were analyzed, following the pretreatment of cells with BDMC and the co-culture with rotenone.

The results of the cell viability assay revealed that compared with the control group, the cell survival rate was decreased in response to the treatment with $100 \mathrm{nM}$ rotenone. Notably, compared with the rotenone-treated cells, BDMC treatment significantly increased the cell survival rate in a dose-dependent manner (Fig. 1A).

It was further discovered that compared with the control group, BDMC treatment did not increase the SOD levels (Fig. 1B), while the SOD levels were decreased in response to the treatment with rotenone. Moreover, compared with the rotenone-induced group, BDMC treatment significantly increased the SOD levels (Fig. 1B). A similar trend was observed for the GSH levels (Fig. 1C).

\section{BDMC treatment enhances the phosphorylation levels of JAK/STAT3 in SH-SY5Y cells treated with rotenone}

To investigate the effects of BDMC treatment on rotenone-induced SH-SY5Y cells, the phosphorylation levels of JAK2/STAT3 were evaluated following the pretreatment of cells with BDMC and the co-culture with rotenone.

The western blotting results revealed that compared with the control group (i.e. rotenone-treated cells), BDMC treatment did not upregulate the JAK2 and STAT3 phosphorylation levels. The JAK2 and STAT3 phosphorylation levels were downregulated in response to the treatment with rotenone. Moreover, compared with the rotenone-induced group, BDMC treatment significantly upregulated the JAK2 and STAT3 phosphorylation levels (Fig. 2A-C).

These results suggested that BDMC may inhibit the toxicity induced by rotenone in SH-SY5Y cells.

\section{AG490, an inhibitor of the JAK/STAT3 signaling pathway, reverses the cell-protective effects of BDMC treatment in SH-SY5Y cells treated with rotenone}

To investigate the effects of the JAK/STAT3 signaling pathway on the cell-protective role of BDMC in rotenone-induced SH-SY5Y cells, STAT3 phosphorylation levels, cell survival, and SOD and GSH activities were evaluated following the pretreatment with AG490 and BDMC, and the co-culture with rotenone.

The results of the cell viability assay demonstrated that BDMC treatment did not increase the cell survival of SH-SY5Y cells induced by rotenone following the inhibition of JAK/STAT3 signaling pathway (Fig. 3A).

In addition, it was observed that BDMC treatment did not increase the SOD levels in SH-SY5Y cells induced by rotenone following the inhibition of JAK/ /STAT3 signaling pathway (Fig. 3B). A similar trend for GSH levels was observed (Fig. 3C).

These results suggested that BDMC treatment may not inhibit the toxicity induced by rotenone in 


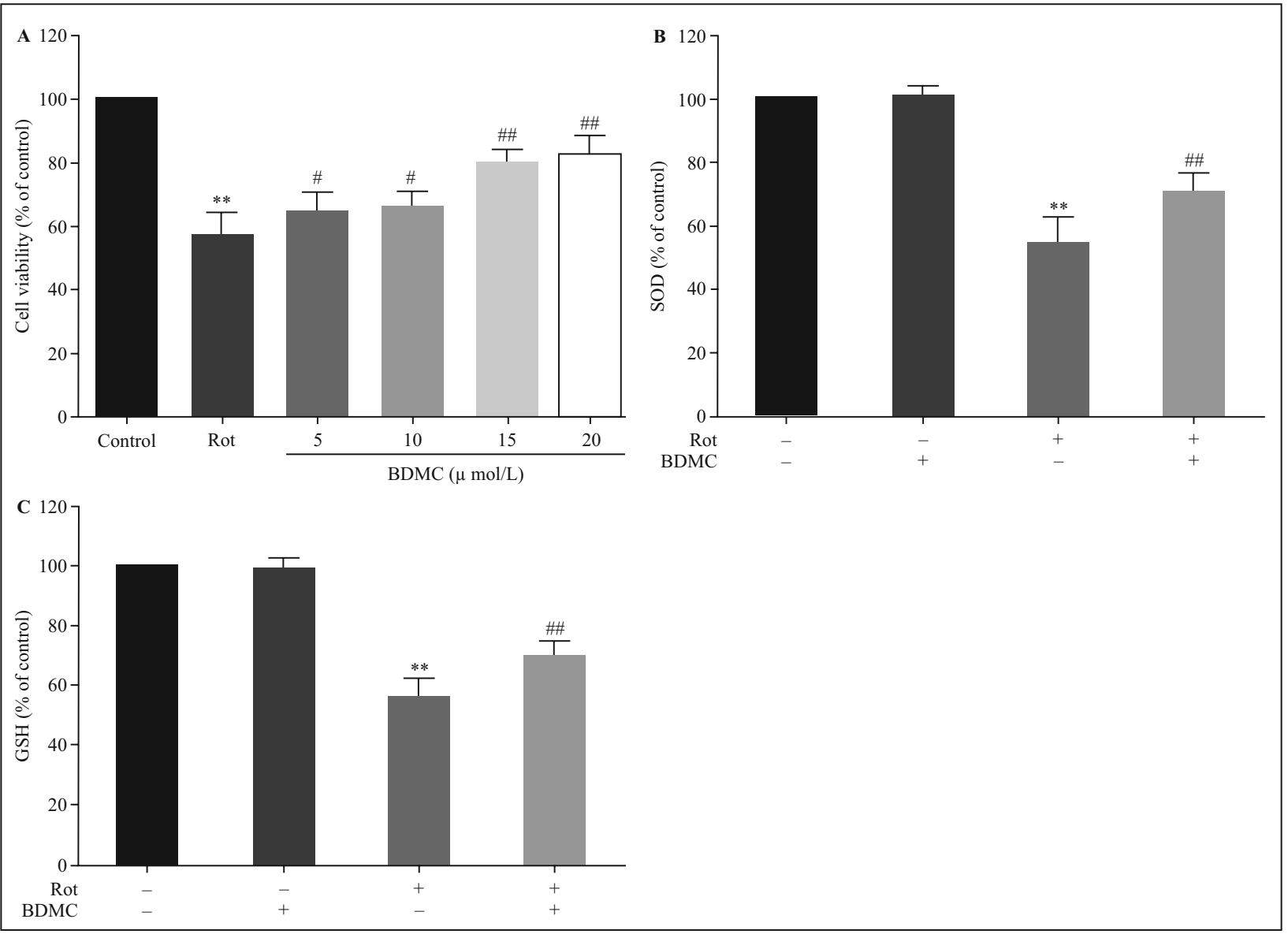

Figure 1. Determination of the effects of BDMC treatment on cell survival and oxidative stress in SH-SY5Y cells treated with rotenone. (A) A total of $1 \times 10^{4} \mathrm{SH}-\mathrm{SY} 5 \mathrm{Y}$ cells were pretreated with $\mathrm{BDMC}(0,5,10,15$ or $20 \mu \mathrm{M})$ for 30 min prior to a 24-h co-culture with $100 \mathrm{nM}$ rotenone, and the cell survival rate was calculated. A total of $1 \times 10^{4} \mathrm{SH}-\mathrm{SY} 5 \mathrm{Y}$ cells were pretreated with $15 \mu \mathrm{M}$ BDMC for $30 \mathrm{~min}$ prior to a $24-\mathrm{h}$ co-culture with $100 \mathrm{nM}$ rotenone, and (B) SOD and (C) GSH activities were measured. ${ }^{* *} \mathrm{P}<0.01$ vs. control; ${ }^{\# \#} \mathrm{P}<0.01$, ${ }^{\#} \mathrm{P}<0.05$ vs. rotenone-induced group from 5 independent experiments.

SH-SY5Y cells upon the inhibition of the JAK/STAT3 signaling pathway.

\section{S3I-201, an inhibitor of the STAT3 signaling pa- thway, reverses the cell-protective effects of BDMC treatment in SH-SY5Y cells treated with rotenone} To investigate the effects of the JAK signaling pathway on the cell-protective role of BDMC in SH-SY5Y cells induced by rotenone, the phosphorylation levels of JAK, cell survival, and SOD and GSH activities were analyzed following the pretreatment of cells with S3I-201 and BDMC and the co-culture with rotenone.

The results of the cell viability assay revealed that BDMC treatment did not increase the cell survival of SH-SY5Y cells induced by rotenone following the inhibition of the JAK signaling pathway (Fig. 4A).

Furthermore, BDMC treatment did not increase the SOD levels in rotenone-induced SH-SY5Y cells follow- ing the inhibition of the JAK signaling pathway (Fig. 4B). A similar pattern was observed for GSH levels (Fig. 4C).

These results indicated that BDMC treatment may not inhibit the toxicity induced by rotenone in SH-SY5Y cells upon the inhibition of the JAK signaling pathway.

\section{Discussion}

In the present study, BDMC treatment was revealed to enhance the cell survival, antioxidative stress ability and the phosphorylation levels of JAK/STAT3 in SH-SY5Y cells treated with rotenone. Moreover, following the inhibition of the JAK/STAT3 signaling pathway, BDMC was unable to exert these cell-protective roles. Thus, the present study suggested that BDMC treatment may protect SH-SY5Y cells from the neurotoxicity induced by rotenone treatment via the JAK/STAT3 signaling pathway. 


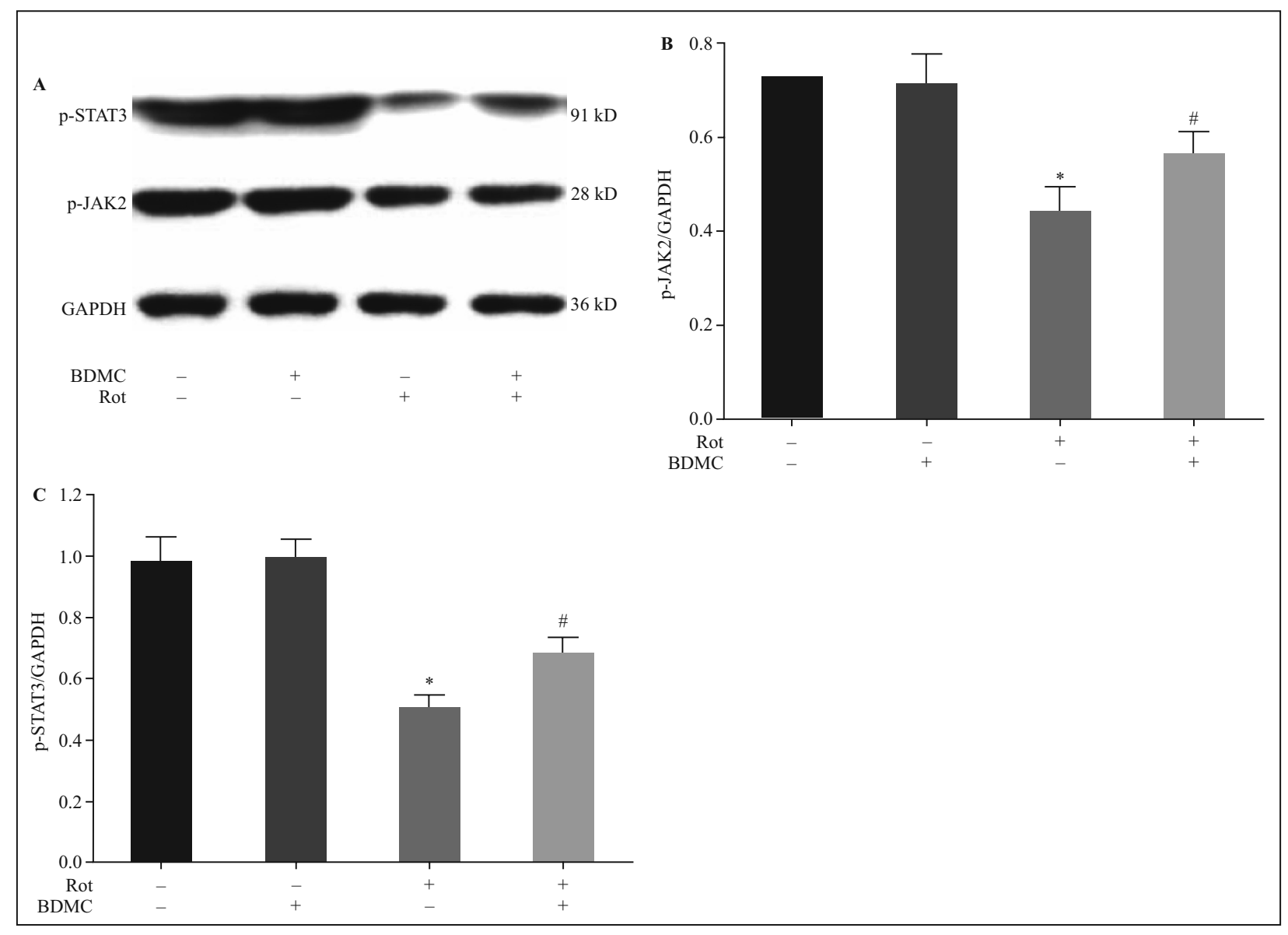

Figure 2. Determination of the effects of BDMC treatment on the JAK/STAT3 signaling pathway in SH-SY5Y cells treated with rotenone. (A) A total of $1 \times 10^{4} \mathrm{SH}-\mathrm{SY} 5 Y$ cells were pretreated with $15 \mu \mathrm{M}$ BDMC for 30 min prior to a 24 -h co-culture with $100 \mathrm{nM}$ rotenone, and western blotting was performed. Representative western blots are shown. The phosphorylation levels of (B) JAK2 and (C) STAT3 were upregulated following the treatment with BDMC. *P $<0.05$ vs. control group; ${ }^{\#} \mathrm{P}<0.05$ vs. rotenone-induced group from 5 independent experiments.

PD is a slow, progressive neurodegenerative disease that is associated with the degeneration of dopaminergic neurons [28]. The loss of dopamine accounts for numerous of the symptoms that accompany the disease, including motor dysfunction, mood alterations and cognitive impairment [29]. Due to difficulties in studying the progression of PD in humans, the treatment of the catecholaminergic neuroblastoma cell line SH-SY5Y with various neurotoxins has been widely used to construct a model closely mimicking $\mathrm{PD}$ in vitro [30-32]. In the present study, BDMC treatment was discovered to decrease the cell death of SH-SY5Y cells induced by rotenone.

Oxidative stress has been identified to account for the progressive death of the dopaminergic neurons [33]. The apoptotic death of nigrostriatal dopaminergic neurons is initiated by oxidative stress [34]. SOD and GSH are important antioxidants that scavenge oxygen free radicals, thus protecting against oxidative stress. In PD model rats, the levels of GSH, SOD and other free radical scavenging enzymes in the brain of the model rats were significantly reduced, further indicating that the rat model of PD demonstrated a reduced ability to scavenge oxygen free radicals; this led to the accumulation of ROS in the tissue cells, which in turn induced the continuous progression of PD [35]. In the present study, BDMC was discovered to protect against oxidative stress.

The activation of the JAK2/STAT3 signaling pathway serves as a protective mechanism against oxidative stress [36]. In the present study, the phosphorylation levels of the JAK2/STAT3 signaling pathway were identified to be upregulated in response to BDMC treatment, whereas following the inhibition of this pathway, BDMC was unable to exert its functional roles.

In conclusion, the findings of the present study suggested that BDMC treatment may alleviate the 


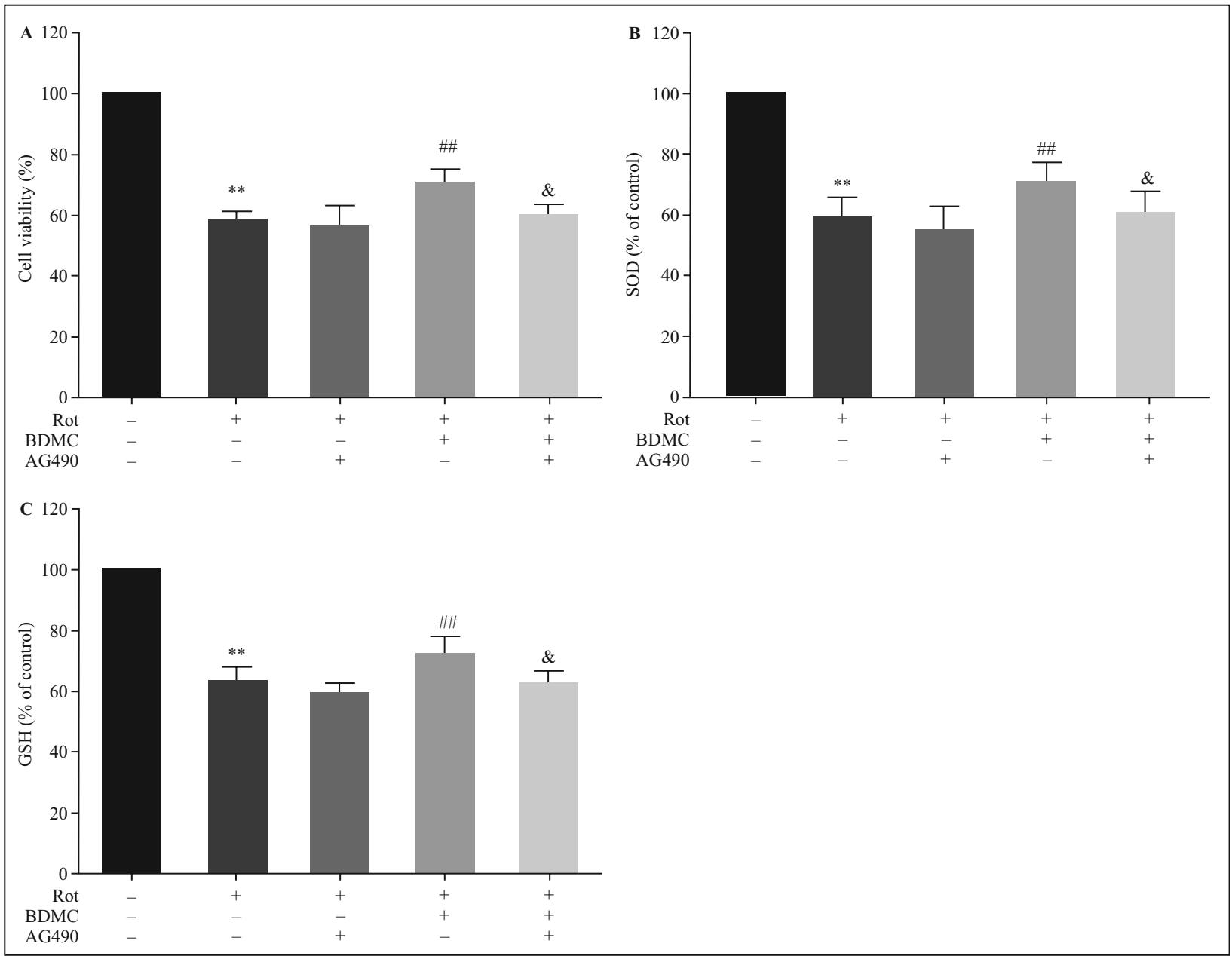

Figure 3. Determination of the effects of BDMC treatment on the cell survival and oxidative stress in SH-SY5Y cells treated with rotenone following the inhibition of the JAK/STAT3 signaling pathway. A total of $1 \times 10^{4} \mathrm{SH}-\mathrm{SY} 5 \mathrm{Y}$ cells were co-pretreated with $10 \mu \mathrm{M}$ AG490 (an inhibitor of the JAK/STAT3 signaling pathway) and $15 \mu \mathrm{M}$ BDMC for 30 min prior to a 24-h co-culture with $100 \mathrm{nM}$ rotenone. The (A) cell survival rate, (B) SOD activity and (C) GSH activity were not increased in response to the treatment with BDMC following the inhibition of the JAK/STAT3 signaling pathway. $* * \mathrm{P}<0.01 v s$. control group; ${ }^{\# \#} \mathrm{P}<0.01,{ }^{\#} \mathrm{P}<0.05$ vs. rotenone-induced group; ${ }^{\mathrm{P}}<0.05$ vs. BDMC + rotenone-induced group from 5 independent experiments.

neurotoxicity of rotenone in SH-SY5Y cells by improving cell survival and exerting antioxidative stress properties. These effects were indicated to occur through the inhibition of the JAK/STAT3 signaling pathway, which may provide a foundation for BDMC treatment to be considered as a novel therapeutic strategy for the treatment of PD.

\section{Acknowledgements}

We thank the Hunan Provincial Natural Science

Foundation of China (grant nos. 2019JJ40267, None.
2017JJ3273, 2018JJ2358), Hunan Health Commission project (grant nos. 20201911, 20200469, 20201963, C20180238) and Hengyang Science and technology project (grant no.2016KJ38) for support.

\section{Declaration of competing interest}

All authors declare no competing interests.

\section{Disclosure of conflict of interest}




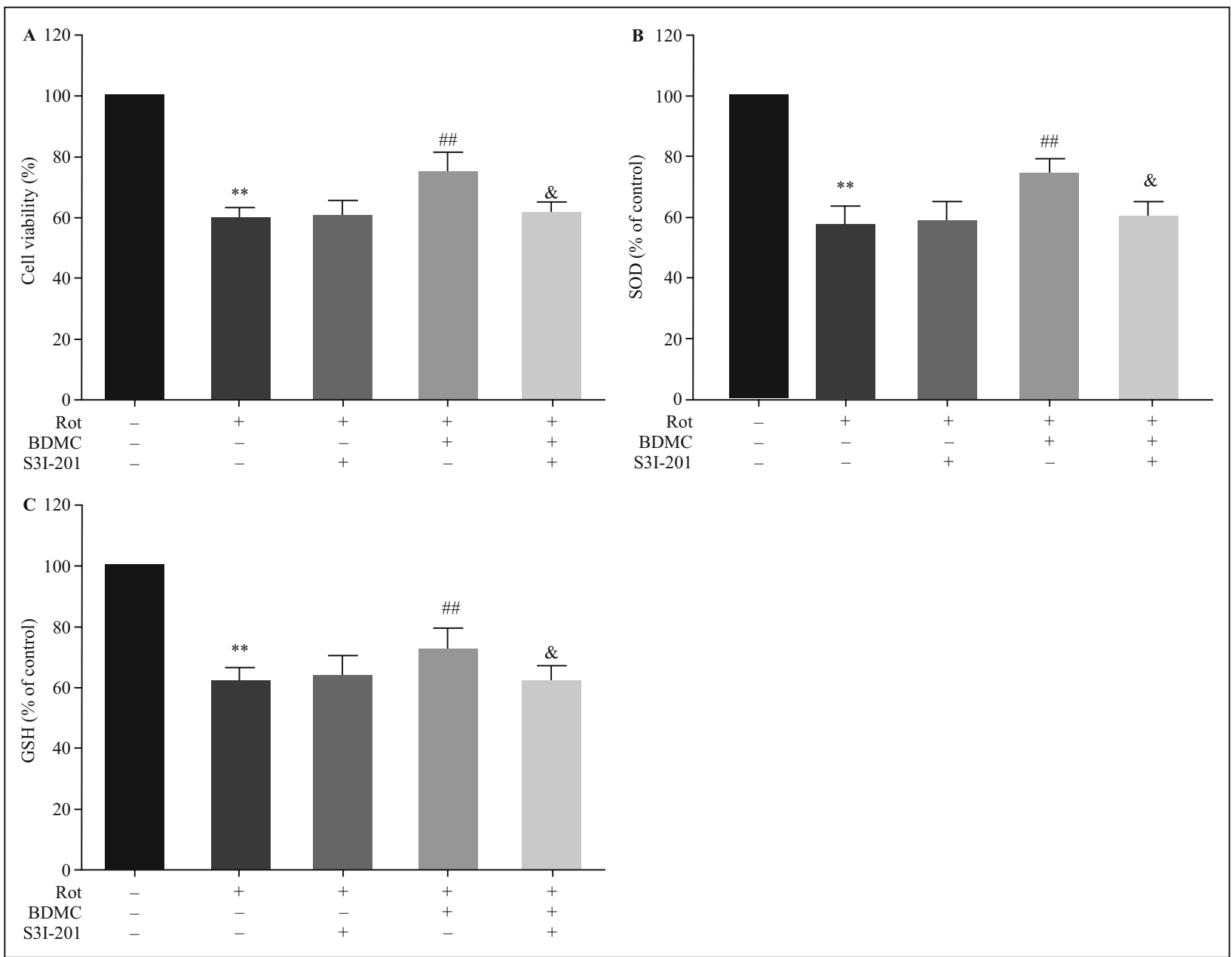

Figure 4. Determination of the effects of BDMC treatment on the cell survival and oxidative stress in SH-SY5Y cells treated with rotenone following the inhibition of the STAT3 signaling pathway. A total of $1 \times 10^{4} \mathrm{SH}-\mathrm{SY} 5 \mathrm{Y}$ cells were co-pretreated with $100 \mathrm{nM}$ S3I-201 (an inhibitor of the STAT3 signaling pathway) and $15 \mu \mathrm{M}$ BDMC for 30 min prior to a 24-h co-culture with $100 \mathrm{nM}$ rotenone. The (A) cell survival rate, $(\mathbf{B})$ SOD activity and $(\mathbf{C}) \mathrm{GSH}$ activity were not increased in response to the treatment with BDMC upon the inhibition of the STAT3 signaling pathway. ${ }^{* *} \mathrm{P}<0.01$ vs. control group; ${ }^{\# \#} \mathrm{P}<0.01$, ${ }^{\#} \mathrm{P}<0.05$ vs. rotenone-induced group; ${ }^{\&} \mathrm{P}<0.05$ vs. BDMC + rotenone-induced group from 5 independent experiments.

\section{References}

1. Cao Xu, Cao L, Ding L, et al. A New Hope for a Devastating Disease: Hydrogen Sulfide in Parkinson's Disease. Mol Neurobiol. 2018; 55(5): 3789-3799, doi: 10.1007/s12035-0170617-0, indexed in Pubmed: 28536975.

2. Keeney PM, Xie J, Capaldi RA, et al. Parkinson's disease brain mitochondrial complex I has oxidatively damaged subunits and is functionally impaired and misassembled. J Neurosci. 2006; 26(19): 5256-5264, doi: 10.1523/JNEUROSCI.0984-06.2006, indexed in Pubmed: 16687518.

3. Chaudhuri K, Schapira A. Non-motor symptoms of Parkinson's disease: dopaminergic pathophysiology and treatment. The Lancet Neurology. 2009; 8(5): 464-474, doi: 10.1016/ s1474-4422(09)70068-7.

4. Xia R, Mao ZH. Progression of motor symptoms in Parkinson's disease. Neurosci Bull. 2012; 28(1): 39-48, doi: 10.1007/ s12264-012-1050-z, indexed in Pubmed: 22233888.

5. Mattson MP. Apoptosis in neurodegenerative disorders. Nat Rev Mol Cell Biol. 2000; 1(2): 120-129, doi: 10.1038/35040009, indexed in Pubmed: 11253364.
6. Onyango IG. Mitochondrial dysfunction and oxidative stress in Parkinson's disease. Neurochem Res. 2008; 33(3): 589597, doi: 10.1007/s11064-007-9482-y, indexed in Pubmed: 17940895.

7. Youn JK, Kim DW, Kim ST, et al. PEP-1-HO-1 prevents MPTP-induced degeneration of dopaminergic neurons in a Parkinson's disease mouse model. BMB Rep. 2014; 47(10): 569-574, doi: 10.5483/bmbrep.2014.47.10.286, indexed in Pubmed: 24499676.

8. Gagliardi S, Ghirmai S, Abel KJ, et al. Evaluation in vitro of synthetic curcumins as agents promoting monocytic gene expression related to $\beta$-amyloid clearance. Chem Res Toxicol. 2012; 25(1): 101-112, doi: 10.1021/tx200246t, indexed in Pubmed: 22029407.

9. Sandur SK, Pandey MK, Sung B, et al. Curcumin, demethoxycurcumin, bisdemethoxycurcumin, tetrahydrocurcumin and turmerones differentially regulate anti-inflammatory and anti-proliferative responses through a ROS-independent mechanism. Carcinogenesis. 2007; 28(8): 1765-1773, doi: 10.1093/ carcin/bgm123, indexed in Pubmed: 17522064. 
10. Ramezani M, Hatamipour M, Sahebkar A. Promising anti-tumor properties of bisdemethoxycurcumin: A naturally occurring curcumin analogue. J Cell Physiol. 2018; 233(2): 880-887, doi: 10.1002/jcp.25795, indexed in Pubmed: 28075008.

11. Basile V, Ferrari E, Lazzari S, et al. Curcumin derivatives: molecular basis of their anti-cancer activity. Biochem Pharmacol. 2009; 78(10): 1305-1315, doi: 10.1016/j.bcp.2009.06.105, indexed in Pubmed: 19580791.

12. Jenner P. Oxidative stress in Parkinson's disease. Ann Neurol. 2003; 53 Suppl 3: S26-36; discussion S36, doi: 10.1002/ ana.10483, indexed in Pubmed: 12666096.

13. Reynolds A, Laurie C, Mosley RL, et al. Oxidative Stress and the Pathogenesis of Neurodegenerative Disorders. Int Rev Neurobiol. 2007: 297-325, doi: 10.1016/s0074-7742(07)820162, indexed in Pubmed: 17678968.

14. La Fortezza M, Schenk M, Cosolo A, et al. JAK/STAT signalling mediates cell survival in response to tissue stress. Development. 2016; 143(16): 2907-2919, doi: 10.1242/dev.132340, indexed in Pubmed: 27385008.

15. Hoffmann CJ, Harms U, Rex A, et al. Vascular signal transducer and activator of transcription-3 promotes angiogenesis and neuroplasticity long-term after stroke. Circulation. 2015; 131(20): 1772-1782, doi: 10.1161/CIRCULATIONAHA.114.013003, indexed in Pubmed: 25794850.

16. Eum WS, Shin MJ, Lee CH, et al. Neuroprotective effects of Tat-ATOX1 protein against MPP-induced SH-SY5Y cell deaths and in MPTP-induced mouse model of Parkinson's disease. Biochimie. 2019; 156: 158-168, doi: 10.1016/j.biochi.2018.10.010, indexed in Pubmed: 30352250.

17. Park SI, Lee EH, Kim SoRa, et al. Anti-apoptotic effects of Curcuma longa L. extract and its curcuminoids against blue light-induced cytotoxicity in A2E-laden human retinal pigment epithelial cells. J Pharm Pharmacol. 2017; 69(3): 334 -340, doi: 10.1111/jphp.12691, indexed in Pubmed: 28155996.

18. Im JH, Yeo InJ, Hwang CJu, et al. PEGylated Erythropoietin Protects against Brain Injury in the MCAO-Induced Stroke Model by Blocking NF- B Activation. Biomol Ther (Seoul). 2020; 28(2): 152-162, doi: 10.4062/biomolther.2019.147, indexed in Pubmed: 31813204

19. Chen S, Hou Y, Zhao Z, et al. Neuregulin-1 Accelerates Functional Motor Recovery by Improving Motoneuron Survival After Brachial Plexus Root Avulsion in Mice. Neuroscience. 2019; 404: 510-518, doi: 10.1016/j.neuroscience.2019.01.054, indexed in Pubmed: 30731156.

20. Li J, Chen S, Zhao Z, et al. Effect of VEGF on Inflammatory Regulation, Neural Survival, and Functional Improvement in Rats following a Complete Spinal Cord Transection. Front Cell Neurosci. 2017; 11: 381, doi: 10.3389/fncel.2017.00381, indexed in Pubmed: 29238292.

21. Chen SX, He JH, Mi YJ, et al. A mimetic peptide of 2,6-sialyllactose promotes neuritogenesis. Neural Regen Res. 2020; 15(6): 1058-1065, doi: 10.4103/1673-5374.270313, indexed in Pubmed: 31823885.

22. Yuhai GU, Zhen Z. Significance of the changes occurring in the levels of interleukins, SOD and MDA in rat pulmonary tissue following exposure to different altitudes and exposure times. Exp Ther Med. 2015; 10(3): 915-920, doi: 10.3892/ etm.2015.2604, indexed in Pubmed: 26622414.

23. Mao GX, Zheng LD, Cao YB, et al. Antiaging effect of pine pollen in human diploid fibroblasts and in a mouse model induced by D-galactose. Oxid Med Cell Longev. 2012;
2012: 750963, doi: 10.1155/2012/750963, indexed in Pubmed: 22577492.

24. Zhang Bo, Chen $\mathrm{Na}$, Chen $\mathrm{H}$, et al. The critical role of redox homeostasis in shikonin-induced HL-60 cell differentiation via unique modulation of the Nrf2/ARE pathway. Oxid Med Cell Longev. 2012; 2012: 781516, doi: 10.1155/2012/781516, indexed in Pubmed: 23119122.

25. Xu J, Hu C, Chen S, et al. Neuregulin-1 protects mouse cerebellum against oxidative stress and neuroinflammation. Brain Res. 2017; 1670: 32-43, doi: 10.1016/j.brainres.2017.06.012, indexed in Pubmed: 28623147.

26. Chen $\mathrm{Sx}, \mathrm{Hu} \mathrm{Cl}$, Liao Yh, et al. L1 modulates PKD1 phosphorylation in cerebellar granule neurons. Neurosci Lett. 2015; 584: 331-336, doi: 10.1016/j.neulet.2014.11.012, indexed in Pubmed: 25445362.

27. Jiang Q, Chen S, Hu C, et al. Neuregulin-1 (Nrg1) signaling has a preventive role and is altered in the frontal cortex under the pathological conditions of Alzheimer's disease. Mol Med Rep. 2016; 14(3): 2614-2624, doi: 10.3892/mmr.2016.5542, indexed in Pubmed: 27486021.

28. Vogt Weisenhorn DM, Giesert F, Wurst W. Diversity matters - heterogeneity of dopaminergic neurons in the ventral mesencephalon and its relation to Parkinson's Disease. J Neurochem. 2016; 139 Suppl 1: 8-26, doi: 10.1111/jnc.13670, indexed in Pubmed: 27206718.

29. Olanow CW, Schapira AHV, Agid Y. Neuroprotection for Parkinson's disease: prospects and promises. Ann Neurol. 2003; 53 Suppl 3: S1-S2, doi: 10.1002/ana.10566, indexed in Pubmed: 12666093.

30. Xie Hr, Hu Ls, Li Gy. SH-SY5Y human neuroblastoma cell line: in vitro cell model of dopaminergic neurons in Parkinson's disease. Chin Med J (Engl). 2010; 123(8): 1086-1092, indexed in Pubmed: 20497720.

31. Xicoy H, Wieringa Bé, Martens GJM. The SH-SY5Y cell line in Parkinson's disease research: a systematic review. Mol Neurodegener. 2017; 12(1): 10, doi: 10.1186/s13024-017-0149-0, indexed in Pubmed: 28118852.

32. Morales-Garcia JA, Aguilar-Morante D, Hernandez-Encinas E, et al. Silencing phosphodiesterase 7B gene by lentiviral-shRNA interference attenuates neurodegeneration and motor deficits in hemiparkinsonian mice. Neurobiol Aging. 2015; 36(2): 1160-1173, doi: 10.1016/j.neurobiolaging.2014.10.008, indexed in Pubmed: 25457552.

33. Dexter DT, Jenner P. Parkinson disease: from pathology to molecular disease mechanisms. Free Radic Biol Med. 2013; 62: 132-144, doi: 10.1016/j.freeradbiomed.2013.01.018, indexed in Pubmed: 23380027.

34. Agrawal S, Singh A, Tripathi P, et al. Cypermethrin-induced nigrostriatal dopaminergic neurodegeneration alters the mitochondrial function: a proteomics study. Mol Neurobiol. 2015; 51(2): 448-465, doi: 10.1007/s12035-014-8696-7, indexed in Pubmed: 24760363.

35. Lee HJ, Shin SY, Choi C, et al. Formation and removal of alpha-synuclein aggregates in cells exposed to mitochondrial inhibitors. J Biol Chem. 2002; 277(7): 5411-5417, doi: 10.1074/ jbc.M105326200, indexed in Pubmed: 11724769.

36. Zheng Wx, Wang F, Cao Xl, et al. Baicalin protects PC-12 cells from oxidative stress induced by hydrogen peroxide via anti-apoptotic effects. Brain Inj. 2014; 28(2): 227-234, doi: 10.3109/02699052.2013.860469, indexed in Pubmed: 24456060 . 